\title{
Nonlinear Fuzzy Model Predictive Control for a PWR Nuclear Power Plant
}

\author{
Xiangjie Liu and Mengyue Wang \\ State Key Laboratory of Alternate Electrical Power System with Renewable Energy Sources, \\ North China Electric Power University, Beijing 102206, China
}

Correspondence should be addressed to Xiangjie Liu; liuxj@ncepu.edu.cn

Received 22 March 2014; Accepted 7 May 2014; Published 2 June 2014

Academic Editor: Chengjin Zhang

Copyright (C) 2014 X. Liu and M. Wang. This is an open access article distributed under the Creative Commons Attribution License, which permits unrestricted use, distribution, and reproduction in any medium, provided the original work is properly cited.

Reliable power and temperature control in pressurized water reactor (PWR) nuclear power plant is necessary to guarantee high efficiency and plant safety. Since the nuclear plants are quite nonlinear, the paper presents nonlinear fuzzy model predictive control (MPC), by incorporating the realistic constraints, to realize the plant optimization. T-S fuzzy modeling on nuclear power plant is utilized to approximate the nonlinear plant, based on which the nonlinear MPC controller is devised via parallel distributed compensation (PDC) scheme in order to solve the nonlinear constraint optimization problem. Improved performance compared to the traditional PID controller for a TMI-type PWR is obtained in the simulation.

\section{Introduction}

In nuclear power plants, steam energy is produced in the nuclear reactor from continuous fission of the atoms of the fuel. The steam is then used to drive the turbine and generator to produce electricity.

Since burning fossil fuels in thermal power plant can have severe environment problem, the development of nuclear power plants should be encouraged. For example in China, although the supercritical and ultrasupercritical technique, as well as integrated gasification combined cycle (IGCC) plants, have been well developed, the coal consumption still reaches about 3.91 million tons in 2012, which comprised over $70 \%$ of national primary energy. Fine particles in the air measuring less than 2.5 micrometres reached 993 micrograms per cubic meter in Beijing on 12 January 2013, compared with the limit of 25 published by the WHO guidelines.

Since nuclear power plants are the complex and nonlinear systems, it is a great challenge to control the power and temperature of the nuclear reactor, especially when widerange power variations occur in the load following condition. Thus, various advanced control schemes have appeared during the past two decades, for example, the observerbased optimal state feedback assisted control [1, 2], the linear quadratic Gaussian with loop transfer recovery control
$[3,4]$, the nonlinear control approach $[5,6]$, and the neural network/fuzzy approaches $[7,8]$.

Modern nuclear power plants should respond to the load demand on the power grid, which demand high plant operation performance, subject to various kinds of constraints. Meanwhile, nuclear safety and radioactive pollution prevention have long been much concerned problem. It is therefore extremely important to reach the economical and safe operation to maximize the thermal efficiency of the nuclear power plant. Under these aims, model predictive control (MPC) shows its obvious advantage, since it is an advanced model-based control scheme. It performs an optimization procedure to calculate optimal control actions at every sampling period based on an explicit process model subject to process input, output, and state constraints. So far, MPC has been well constituted for thermal power plant control [9-11] and also in nuclear power plant water-level control [12-14]. Paper [15] reports a MPC application in a three-dimensional nuclear reactor analysis code for nuclear reactor power.

Nuclear power plants are generally nonlinear, due to the frequent changes of the operating point right across the whole operation range. In general, the nonlinear model predictive control (NMPC) also online solves an optimization problem, by using the sequential quadratic program (SQP). 
The resulting nonlinear programming problems are usually nonconvex, and the online computational burden is generally large. Since the nonlinearities vary with operating power levels, as well as ageing effects $[5,16]$, fuzzy models are used to express the plant dynamic. MPCs are realized in a parallel distributed compensation (PDC) scheme; that is, for each local model, a MPC is designed. The overall controller, which is nonlinear in nature, is similarly constructed by combining local controllers via fuzzy inference. Simulations on the three-mile island- (TMI-) type pressurized water reactor (PWR) show the effectiveness of the proposed method.

\section{The Plant Description}

As shown in Figure 1, nuclear fission reaction in the reactor releases tremendous energy, which is transferred from the reactor to the steam generator by the coolant, heating the water in the steam generator to generate abundant steam. Then the turbine is driven by steam to generate electricity. The main objective is to control the power and temperature of the reactor by inserting or elevating the control rods. Conventionally, reactor control system controls the average temperature of the reactor core coolant to track the reference temperature which is proportional to the turbine load, in order to guarantee well matching between the reactor power level and the load demand at the same time. This temperature control strategy, considering not only high thermal efficiency but also technical limits and economical and safe operation, is widely used in modern PWR power plants.

However, the plant is complex and highly nonlinear whose parameters vary with the operation conditions. Meanwhile, realistic constraints in the system may lead to actuator saturation. The performance of the conventional controllers is often unsatisfactory. Besides, unpredictable disturbances in the temperature measurement system will induce fluctuations on the measuring values of coolant temperature, which may lead to the abnormal control rod action and thus must be considered in the controller design. The proposed TakagiSugeno fuzzy modeling method is used to approximate the nonlinear plant, based on which the nonlinear MPC controller is devised via PDC scheme in order to solve the nonlinear and constraint problems in the reactor power and temperature control system.

\section{T-S Fuzzy Modeling}

The reactor model considered is the point kinetics with one delayed neutron group and also coolant and fuel temperature feedback. It is typical for a TMI-type PWR at the middle of the fuel cycle rated at $2500 \mathrm{MW}[2,7]$ :

$$
\begin{aligned}
& \frac{d}{d t} n_{r}=\frac{\delta \rho-\beta}{\Lambda} n_{r}+\lambda c_{r}, \\
& \frac{d}{d t} c_{r}=\lambda n_{r}-\lambda c_{r},
\end{aligned}
$$

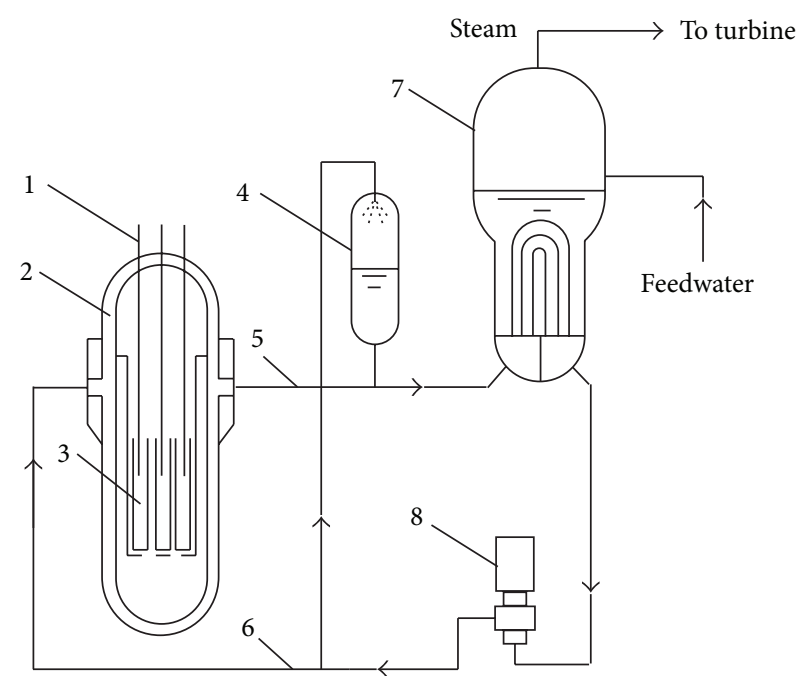
(1) Control rod
(5) Hot leg
(2) Containment shell
(3) Reactor core
(6) Cold leg
(7) Steam generator
(4) Pressurizer
(8) Reactor coolant pump

FIGURE 1: PWR and steam generation process.

$$
\begin{gathered}
\frac{d}{d t} T_{f}=\frac{f_{f} P_{a 0}}{\mu_{f}} n_{r}-\frac{\Omega}{\mu_{f}} T_{f} \\
+\frac{\Omega}{2 \mu_{f}} T_{l}+\frac{\Omega}{2 \mu_{f}} T_{e}, \\
\frac{d}{d t} T_{l}=\frac{\left(1-f_{f}\right) P_{a 0}}{\mu_{c}} n_{r}+\frac{\Omega}{\mu_{c}} T_{f} \\
\quad-\frac{(2 M+\Omega)}{2 \mu_{c}} T_{l}+\frac{(2 M-\Omega)}{2 \mu_{c}} T_{e}, \\
\frac{d}{d t} \delta \rho_{r}=G_{r} z_{r}, \\
\delta \rho=\delta \rho_{r}+\alpha_{f}\left(T_{f}-T_{f 0}\right)+\frac{\alpha_{c}}{2}\left(T_{l}-T_{l 0}\right) \\
+\frac{\alpha_{c}}{2}\left(T_{e}-T_{e 0}\right) .
\end{gathered}
$$

The symbols in the above equations are demonstrated in the Nomenclature section.

Define

$$
\begin{gathered}
\delta n_{r}=n_{r}-n_{r 0}, \\
\delta c_{r}=c_{r}-c_{r 0}, \\
\delta T_{f}=T_{f}-T_{f 0}, \\
\delta T_{l}=T_{l}-T_{l 0}, \\
\delta \rho_{r}=\rho_{r}-\rho_{r 0},
\end{gathered}
$$


where the symbol $\delta$ indicates a deviation about an equilibrium point. $n_{r 0}, c_{r 0}, T_{f 0}, T_{l 0}$, and $\rho_{r 0}$ correspond to the values of $n_{r}, c_{r}, T_{f}, T_{l}$, and $\rho_{r}$ at an equilibrium point, respectively. follows:

Choose the state, input, and output vector, respectively, as

$$
\begin{aligned}
& \mathbf{x}=\left[\begin{array}{lllll}
\delta n_{r} & \delta c_{r} & \delta T_{f} & \delta T_{l} & \delta \rho_{r}
\end{array}\right]^{T}, \\
& u=z_{r} \text {, } \\
& \mathbf{y}=\left[\begin{array}{ll}
\delta n_{r} & \delta T_{l}
\end{array}\right]^{T} \text {. }
\end{aligned}
$$

The model can be linearized at the equilibrium point using the perturbation theory which is valid only for $\delta n_{r} \ll$ $n_{r 0}$. The linear model can be written as the following state space form:

$$
\begin{gathered}
\dot{\mathbf{x}}=\mathbf{A x}+\mathbf{B} u, \\
\mathbf{y}=\mathbf{C} \mathbf{x} .
\end{gathered}
$$

The corresponding matrices $\mathbf{A}, \mathbf{B}$, and $\mathbf{C}$, are

$$
\begin{aligned}
& \mathbf{A}=\left[\begin{array}{ccccc}
-\frac{\beta}{\Lambda} & \frac{\beta}{\Lambda} & \frac{n_{r 0}^{i} \alpha_{f}}{\Lambda} & \frac{n_{r 0}^{i} \alpha_{c}}{2 \Lambda} & \frac{n_{r 0}^{i}}{\Lambda} \\
\lambda & -\lambda & 0 & 0 & 0 \\
\frac{f_{f} P_{0 a}}{\mu_{f}} & 0 & -\frac{\Omega}{\mu_{f}} & \frac{\Omega}{2 \mu_{f}} & 0 \\
\frac{\left(1-f_{f}\right) P_{0 a}}{\mu_{c}} & 0 & \frac{\Omega}{\mu_{c}} & -\frac{2 M+\Omega}{2 \mu_{c}} & 0 \\
0 & 0 & 0 & 0 & 0
\end{array}\right], \\
& \mathbf{B}=\left[\begin{array}{lllll}
0 & 0 & 0 & 0 & G_{r}^{i}
\end{array}\right]^{T}, \\
& \mathbf{C}=\left[\begin{array}{lllll}
1 & 0 & 0 & 0 & 0 \\
0 & 0 & 0 & 1 & 0
\end{array}\right] \text {, }
\end{aligned}
$$

where where $n_{r 0}^{i}$ is the power level and $G_{r}^{i}$ is the control rod worth for the $i$ th $(i=1,2, \ldots, 9)$ operating point which is defined in Table 1. The linear model (4) is defined at each operating point $\left(n_{r 0}^{i}, G_{r}^{i}\right)$ and the parameters in (6) are functions of the operating point $[7,17]$. The remaining constant parameters are shown in Table 2. Consider the following:

$$
\begin{aligned}
& \alpha_{f}\left(n_{r 0}\right)=\left(n_{r 0}-4.24\right) \times 10^{-5} \frac{\delta k}{k} /{ }^{\circ} \mathrm{C}, \\
& \alpha_{c}\left(n_{r 0}\right)=\left(-4.0 n_{r 0}-17.3\right) \times 10^{-5} \frac{\delta k}{k} /{ }^{\circ} \mathrm{C}, \\
& \mu_{c}\left(n_{r 0}\right)=\left(\frac{160}{9} n_{r 0}+54.022\right) \mathrm{MW} /{ }^{\circ} \mathrm{C}, \\
& \Omega\left(n_{r 0}\right)=\left(\frac{5}{3} n_{r 0}+4.9333\right) \mathrm{MW} /{ }^{\circ} \mathrm{C}, \\
& M\left(n_{r 0}\right)=\left(28.0 n_{r 0}+74.0\right) \mathrm{MW} /{ }^{\circ} \mathrm{C} .
\end{aligned}
$$

The sampling period is selected to be $1 \mathrm{~s}$, and then nine discrete-time local models of (4) can be described as follows:

$$
\begin{array}{r}
\mathbf{x}(k+1)=\mathbf{G}_{i} \mathbf{x}(k)+\mathbf{H}_{i} u(k), \\
\mathbf{y}(k)=\mathbf{C}_{i} \mathbf{x}(k) \\
\quad(i=1,2, \ldots, 9),
\end{array}
$$

$$
\begin{aligned}
& \mathbf{G}_{1}=\left[\begin{array}{ccccc}
5.42 \times 10^{-1} & 4.51 \times 10^{-1} & -1.40 \times 10^{-2} & -3.59 \times 10^{-2} & 3.75 \times 10^{1} \\
1.12 \times 10^{-3} & 9.99 \times 10^{-1} & -1.16 \times 10^{-5} & -2.97 \times 10^{-5} & 3.09 \times 10^{-2} \\
6.54 \times 10^{-1} & 2.17 \times 10^{-1} & 9.91 \times 10^{-1} & -1.62 \times 10^{-2} & 1.80 \times 10^{1} \\
2.40 \times 10^{-2} & 7.92 \times 10^{-3} & 6.63 \times 10^{-4} & 9.85 \times 10^{-1} & 6.58 \times 10^{-1} \\
0 & 0 & 0 & 0 & 1
\end{array}\right], \\
& \mathbf{G}_{2}=\left[\begin{array}{ccccc}
5.47 \times 10^{-1} & 4.52 \times 10^{-1} & -3.11 \times 10^{-3} & -6.60 \times 10^{-3} & 7.51 \\
1.13 \times 10^{-3} & 9.99 \times 10^{-1} & -2.60 \times 10^{-6} & -5.50 \times 10^{-6} & 6.19 \times 10^{-3} \\
6.56 \times 10^{-1} & 2.17 \times 10^{-1} & 9.97 \times 10^{-1} & -2.22 \times 10^{-3} & 3.61 \\
2.70 \times 10^{-2} & 8.93 \times 10^{-3} & 8.45 \times 10^{-4} & 9.86 \times 10^{-1} & 1.48 \times 10^{-1} \\
0 & 0 & 0 & 0 & 1
\end{array}\right], \\
& \mathbf{G}_{6}=\left[\begin{array}{ccccc}
5.47 \times 10^{-1} & 4.52 \times 10^{-1} & -2.43 \times 10^{-3} & -7.94 \times 10^{-3} & 7.51 \times 10^{1} \\
1.13 \times 10^{-3} & 9.99 \times 10^{-1} & -2.00 \times 10^{-6} & -6.60 \times 10^{-6} & 6.19 \times 10^{-2} \\
6.56 \times 10^{-1} & 2.17 \times 10^{-1} & 9.96 \times 10^{-1} & -2.58 \times 10^{-3} & 3.61 \times 10^{1} \\
2.11 \times 10^{-2} & 6.96 \times 10^{-3} & 8.74 \times 10^{-4} & 9.85 \times 10^{-1} & 1.16 \\
0 & 0 & 0 & 0 & 1
\end{array}\right], \\
& \mathbf{G}_{1}=\mathbf{G}_{4}=\mathbf{G}_{8}, \quad \mathbf{G}_{2}=\mathbf{G}_{3}=\mathbf{G}_{9}, \quad \mathbf{G}_{5}=\mathbf{G}_{6}=\mathbf{G}_{7},
\end{aligned}
$$




$$
\begin{aligned}
& \mathbf{H}_{1}=\left[\begin{array}{l}
2.99 \times 10^{-3} \\
1.60 \times 10^{-6} \\
9.14 \times 10^{-4} \\
3.33 \times 10^{-5} \\
1.45 \times 10^{-4}
\end{array}\right], \quad \mathbf{H}_{4}=\left[\begin{array}{l}
2.89 \times 10^{-4} \\
2.00 \times 10^{-7} \\
8.83 \times 10^{-5} \\
3.60 \times 10^{-6} \\
7.00 \times 10^{-5}
\end{array}\right], \quad \mathbf{H}_{8}=\left[\begin{array}{l}
1.20 \times 10^{-2} \\
6.30 \times 10^{-6} \\
3.66 \times 10^{-3} \\
1.17 \times 10^{-4} \\
2.90 \times 10^{-4}
\end{array}\right], \\
& \mathbf{H}_{1}=\mathbf{H}_{2}=\mathbf{H}_{6}, \quad \mathbf{H}_{3}=\mathbf{H}_{4}=\mathbf{H}_{5}, \quad \mathbf{H}_{7}=\mathbf{H}_{8}=\mathbf{H}_{9} \text {, } \\
& \mathrm{C}_{1}=\left[\begin{array}{lllll}
1 & 0 & 0 & 0 & 0 \\
0 & 0 & 0 & 1 & 0
\end{array}\right], \\
& \mathrm{C}_{1}=\mathrm{C}_{2}=\mathrm{C}_{3}=\mathrm{C}_{4}=\mathrm{C}_{5}=\mathrm{C}_{6}=\mathrm{C}_{7}=\mathrm{C}_{8}=\mathrm{C}_{9} \text {. }
\end{aligned}
$$

TABLE 1: The definition of the operating points.

\begin{tabular}{lccc}
\hline$G_{r}$ & & $n_{r 0}$ & \\
& 0.1 & 0.5 & 1.0 \\
\hline 0.029 & Point 3 & Point 4 & Point 5 \\
0.0145 & Point 2 & Point 1 & Point 6 \\
0.0070 & Point 9 & Point 8 & Point 7 \\
\hline
\end{tabular}

TABLE 2: The constant parameters in the system matrices.

\begin{tabular}{lc}
\hline$\lambda$ & $0.15 \mathrm{~s}^{-1}$ \\
$\beta$ & 0.006019 \\
$f_{f}$ & 0.92 \\
$\Lambda$ & $0.0001 \mathrm{~s}$ \\
$\mu_{f}$ & $23.6 \mathrm{MWs} /{ }^{\circ} \mathrm{C}$ \\
$P_{a 0}$ & $2500.0 \mathrm{MW}$ \\
\hline
\end{tabular}

According to Table 1, power level $n_{r 0}$ and control rod worth $G_{r}$ are selected as the premise variables. $n_{r 0}$ and $G_{r}$ are defined in region $[0,1]$ and region $[0,0.029]$, respectively. $n_{r 0}$ and $G_{r}$ are divided into three fuzzy sets, denoted, respectively, as "low (L)," "medium (M)," and "high (H)." The corresponding univariate membership functions of $n_{r 0}$ or $G_{r}$ are defined by triangle membership functions, as shown in Figure 2. Thus, the space is divided into nine fuzzy subspaces, given as follows:

$$
\begin{aligned}
& R^{(i)}:(i=1,2, \ldots, 9) \\
& \operatorname{IF} z_{1}(k) \text { is } F_{1}^{i} \text { and } z_{2}(k) \text { is } F_{2}^{i} \\
& \operatorname{THEN~} \mathbf{x}_{i}(k+1)=\mathbf{G}_{i} \mathbf{x}_{i}(k)+\mathbf{H}_{i} u_{i}(k) \\
& \mathbf{y}_{i}(k)=\mathbf{C}_{i} \mathbf{x}_{i}(k),
\end{aligned}
$$

where $R^{(i)}$ denotes the $i$ th fuzzy inference rule. $z_{j}(k)(j=$ $1,2)$ are premise variables for $n_{r 0}$ and $G_{r} ; F_{j}^{i}$ denotes the fuzzy set partitioned for the $j$ th premise variable, which is valid in the $i$ th rule. $\left(G_{i}, H_{i}, C_{i}\right)$ represents the consequent $i$ th linear model deduced at the $i$ th equilibrium point in (7).

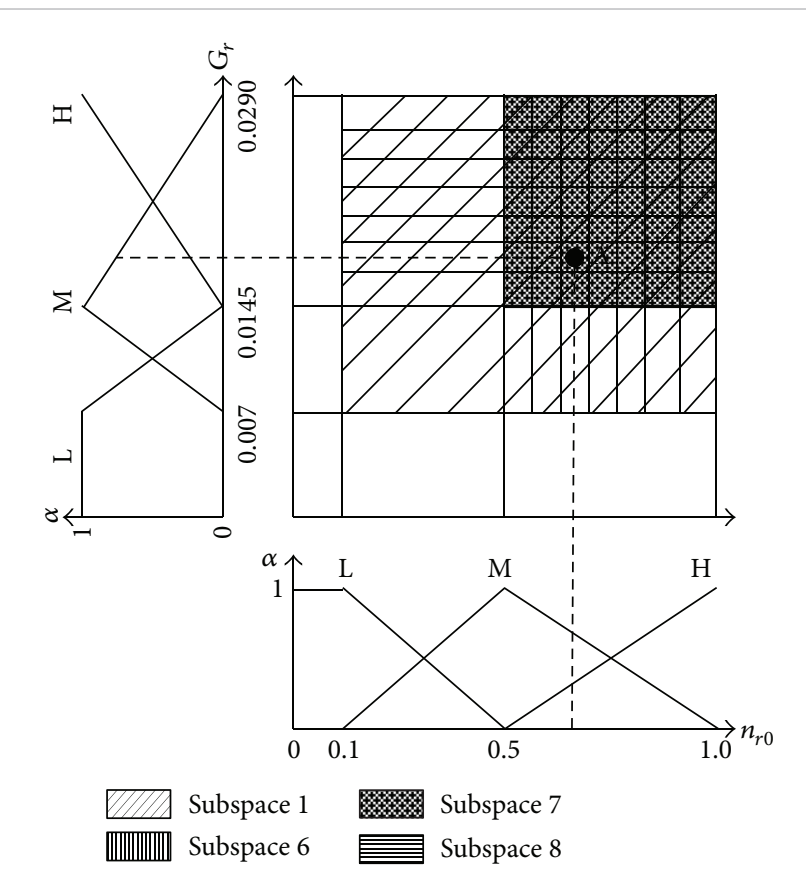

FIGURE 2: The division of fuzzy space and the definition of univariate membership functions.

By using the center of gravity defuzzification method $[10,18]$, the final output of the nonlinear T-S model with nine fuzzy rules is

$$
\begin{gathered}
\mathbf{x}(k+1)=\sum_{i=1}^{9} \bar{\mu}_{i} \mathbf{x}_{i}(k+1)=\sum_{i=1}^{9} \bar{\mu}_{i}\left(\mathbf{G}_{i} \mathbf{x}_{i}(k)+\mathbf{H}_{i} u_{i}(k)\right) \\
\mathbf{y}(k)=\sum_{i=1}^{9} \bar{\mu}_{i} \mathbf{y}_{i}(k)=\sum_{i=1}^{9} \bar{\mu}_{i} \mathbf{C}_{i} \mathbf{x}_{i}(k),
\end{gathered}
$$

where

$$
\begin{aligned}
\bar{\mu}_{i} & =\frac{\mu_{i}}{\sum_{i=1}^{9} \mu_{i}}, \\
\mu_{i} & =\prod_{j=1}^{2} \alpha_{F_{j}^{i}}\left(z_{j}(k)\right) .
\end{aligned}
$$




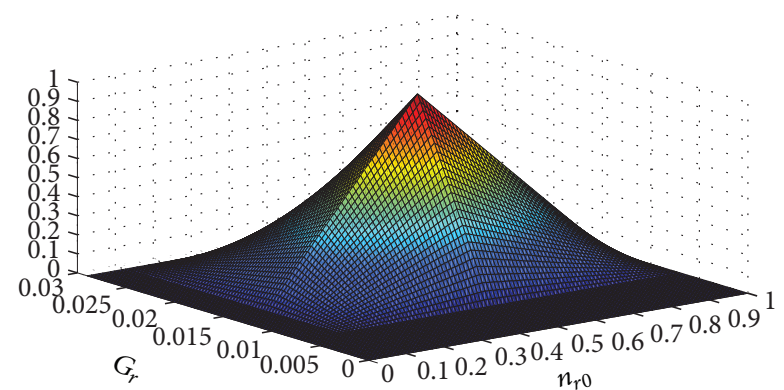

FIGURE 3: Multivariate membership functions in the fuzzy subspace 1.

The $\alpha_{F_{j}^{i}}\left(z_{j}(k)\right)$ denotes the univariate membership function of premise variable $z_{j}(k)$ with respect to the fuzzy set $F_{j}^{i} . \mu_{i}$ is the multivariate membership function obtained by the product of the outputs of the two univariate membership functions, as shown in Figure 3.

Four rules are activated each time instant. At point $\mathrm{A}$ in Figure 2, these four rules are as follows:

$$
\begin{aligned}
& R^{1}: \text { if } G_{r}(k) \text { is } M \text { and } n_{r 0}(k) \text { is } M, \\
& \text { then } \\
& \mathbf{x}_{1}(k+1)=\mathbf{G}_{1} \mathbf{x}_{1}(k)+\mathbf{H}_{1} u_{1}(k) \\
& \mathbf{y}_{1}(k)=\mathbf{C}_{1} \mathbf{x}_{1}(k) . \\
& R^{6}: \text { if } G_{r}(k) \text { is } M \text { and } n_{r 0}(k) \text { is } H, \\
& \text { then } \\
& \mathbf{x}_{6}(k+1)=\mathbf{G}_{6} \mathbf{x}_{6}(k)+\mathbf{H}_{6} u_{6}(k) \\
& \mathbf{y}_{6}(k)=\mathbf{C}_{6} \mathbf{x}_{6}(k) . \\
& R^{7}: \text { if } G_{r}(k) \text { is } H \text { and } n_{r 0}(k) \text { is } H, \\
& \text { then } \\
& \mathbf{x}_{7}(k+1)=\mathbf{G}_{7} \mathbf{x}_{7}(k)+\mathbf{H}_{7} u_{7}(k) \\
& \mathbf{y}_{7}(k)=\mathbf{C}_{7} \mathbf{x}_{7}(k) . \\
& R^{8}: \text { if } G_{r}(k) \text { is } H \text { and } n_{r 0}(k) \text { is } M, \\
& \text { then } \\
& \mathbf{x}_{8}(k+1)=\mathbf{G}_{8} \mathbf{x}_{8}(k)+\mathbf{H}_{8} u_{8}(k) \\
& \mathbf{y}_{8}(k)=\mathbf{C}_{8} \mathbf{x}_{8}(k) .
\end{aligned}
$$

The membership functions are shown in Figure 4.

\section{The Nonlinear Fuzzy MPC}

4.1. The Local Linear MPC. The local linear model (7) can be expressed as follows:

$$
\begin{gathered}
\mathbf{x}(k)=\mathbf{G} \mathbf{x}(k-1)+\mathbf{H} u(k-1), \\
\mathbf{y}(k-1)=\mathbf{C} \mathbf{x}(k-1)
\end{gathered}
$$

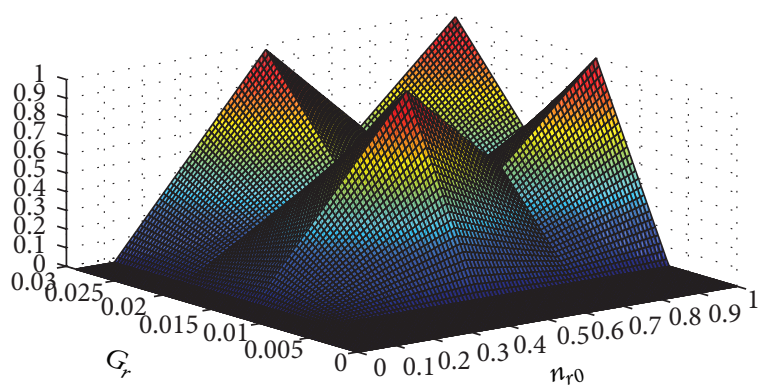

FIgURE 4: Multivariate membership functions activated at point A.

Subtracting (13) from (7) can result in

$$
\begin{gathered}
\Delta \mathbf{x}(k+1)=\mathbf{G} \Delta \mathbf{x}(k)+\mathbf{H} \Delta u(k), \\
\Delta \mathbf{y}(k)=\mathbf{C} \Delta \mathbf{x}(k),
\end{gathered}
$$

where $\Delta \mathbf{x}(k)=\mathbf{x}(k)-\mathbf{x}(k-1), \Delta u(k)=u(k)-u(k-1)$, and $\Delta \mathbf{y}(k)=\mathbf{y}(k)-\mathbf{y}(k-1)$.

Hence, $\Delta \mathbf{y}(k+1)=\mathbf{C} \Delta \mathbf{x}(k+1)=\mathbf{C G} \Delta \mathbf{x}(k)+\mathbf{C H} \Delta u(k)$.

Choosing a new state variable vector $\mathbf{x}^{\prime}(k)=$ $\left[\Delta \mathbf{x}(k)^{T} \quad \mathbf{y}(k)^{T}\right]^{T}$ can result in

$$
\begin{gathered}
{\left[\begin{array}{c}
\Delta \mathbf{x}(k+1) \\
\mathbf{y}(k+1)
\end{array}\right]=\left[\begin{array}{cc}
\mathbf{G} & \mathbf{0} \\
\mathbf{C G} & \mathbf{I}
\end{array}\right]\left[\begin{array}{c}
\Delta \mathbf{x}(k) \\
\mathbf{y}(k)
\end{array}\right]+\left[\begin{array}{c}
\mathbf{H} \\
\mathbf{C H}
\end{array}\right] \Delta u(k)} \\
\mathbf{y}(k)=\left[\begin{array}{ll}
\mathbf{0} & \mathbf{I}
\end{array}\right]\left[\begin{array}{c}
\Delta \mathbf{x}(k) \\
\mathbf{y}(k)
\end{array}\right]
\end{gathered}
$$

Denote (15) as the following normal form:

$$
\mathbf{x}^{\prime}(k+1)=\mathbf{G}^{\prime} \mathbf{x}^{\prime}(k)+\mathbf{H}^{\prime} u(k),
$$$$
\mathbf{y}(k)=\mathbf{C}^{\prime} \mathbf{x}^{\prime}(k),
$$

where $\mathbf{x}^{\prime}(k) \in R^{n^{\prime}}\left(n^{\prime}=n+p\right), \mathbf{G}^{\prime}=\left[\begin{array}{cc}\mathbf{G} & 0 \\ \mathbf{C G} & \mathbf{I}\end{array}\right], \mathbf{H}^{\prime}=\left[\begin{array}{c}\mathbf{H} \\ \mathbf{C H}\end{array}\right]$, and $\mathbf{C}^{\prime}=\left[\begin{array}{ll}\mathbf{0} & \mathbf{I}\end{array}\right] . n$ and $p$ are, respectively, the number of the state variables and the output variables.

The predictive control is obtained by minimizing the following cost function:

$$
J=\left\|\mathbf{Y}-\mathbf{r}_{s}\right\|_{\mathbf{Q}}^{2}+\|\Delta \mathbf{U}\|_{\mathbf{R}}^{2}
$$

The control is obtained by minimizing the cost function at each time instant $k$.

Define

$$
\begin{gathered}
\mathbf{Y}=\left[\widehat{\mathbf{y}}(k+1 \mid k)^{T} \widehat{\mathbf{y}}(k+2 \mid k)^{T} \cdots \widehat{\mathbf{y}}\left(k+N_{P} \mid k\right)^{T}\right]^{T}, \\
\mathbf{r}_{s}=\left[\mathbf{r}(k+1)^{T} \mathbf{r}(k+2)^{T} \cdots \mathbf{r}\left(k+N_{P}\right)^{T}\right]^{T}, \\
\Delta \mathbf{U}=\left[\Delta u(k) \Delta u(k+1) \cdots \Delta u\left(k+N_{c}-1\right)\right]^{T},
\end{gathered}
$$




$$
\begin{gathered}
\mathbf{Q}=\left[\begin{array}{cccc}
\mathbf{Q}(1) & 0 & \cdots & 0 \\
0 & \mathbf{Q}(2) & \cdots & 0 \\
\vdots & \vdots & \ddots & \vdots \\
0 & 0 & \cdots & \mathbf{Q}\left(N_{p}\right)
\end{array}\right], \\
\mathbf{R}=\left[\begin{array}{cccc}
\mathbf{R}(0) & 0 & \cdots & 0 \\
0 & \mathbf{R}(1) & \cdots & 0 \\
\vdots & \vdots & \ddots & \vdots \\
0 & 0 & \cdots & \mathbf{R}\left(N_{c}-1\right)
\end{array}\right],
\end{gathered}
$$

where $\mathbf{Q}$ and $\mathbf{R}$ are the weighting matrices for the prediction error and the control, respectively, $N_{p}$ and $N_{c}$ are, respectively, the costing horizon for the prediction error and the control, and $\widehat{\mathbf{y}}(k+j \mid k)^{T}$ and $\mathbf{r}(k+j)^{T}(j=$ $1,2, \ldots, N_{p}$ ) are the optimum $j$ th step ahead prediction of the system output on data up to time instant $k$ and the $j$ th step ahead reference trajectory, respectively. $\Delta \mathbf{U}$ is the vector composed of the future control increments which is obtained by computing the derivative of $J$, expressed as follows:

$$
\Delta \mathbf{U}=\left(\mathbf{R}+\boldsymbol{\Phi}^{T} \mathbf{Q} \boldsymbol{\Phi}\right)^{-1} \boldsymbol{\Phi}^{T} \mathbf{Q}\left(\mathbf{r}_{s}-\mathbf{F x}(k)\right)
$$

where

$$
\mathbf{F}=\left[\begin{array}{c}
\mathbf{C}^{\prime} \mathbf{G}^{\prime} \\
\mathbf{C}^{\prime} \mathbf{G}^{\prime 2} \\
\mathbf{C}^{\prime} \mathbf{G}^{\prime 3} \\
\vdots \\
\mathbf{C}^{\prime} \mathbf{G}^{\prime N_{p}}
\end{array}\right], \quad \Phi=\left[\begin{array}{ccccc}
\mathbf{C}^{\prime} \mathbf{H}^{\prime} & \mathbf{0} & \mathbf{0} & \cdots & \mathbf{0} \\
\mathbf{C}^{\prime} \mathbf{G}^{\prime} \mathbf{H}^{\prime} & \mathbf{C}^{\prime} \mathbf{H}^{\prime} & \mathbf{0} & \cdots & \mathbf{0} \\
\mathbf{C}^{\prime} \mathbf{G}^{\prime 2} \mathbf{H}^{\prime} & \mathbf{C}^{\prime} \mathbf{G}^{\prime} \mathbf{H}^{\prime} & \mathbf{C}^{\prime} \mathbf{H}^{\prime} & \cdots & \mathbf{0} \\
\vdots & \vdots & \vdots & \ddots & \vdots \\
\mathbf{C}^{\prime} \mathbf{G}^{\prime N_{p}-1} \mathbf{H}^{\prime} & \mathbf{C}^{\prime} \mathbf{G}^{\prime N_{p}-2} \mathbf{H}^{\prime} & \mathbf{C}^{\prime} \mathbf{G}^{\prime N_{p}-3} \mathbf{H}^{\prime} & \cdots & \mathbf{C}^{\prime} \mathbf{G}^{\prime N_{p}-N_{c}} \mathbf{H}^{\prime}
\end{array}\right]
$$

The optimized $N_{c}$ steps ahead control $\Delta \mathbf{U}$ is computed, and only the first step ahead control $\Delta u(k)$ is implemented, using a receding horizon principle [10], giving

$$
\Delta u(k)=\underbrace{\left[\begin{array}{llll}
1 & 0 & \cdots & 0
\end{array}\right]}_{N_{c}} \Delta \mathbf{U} ;
$$

further, $u(k)$ can be obtained by

$$
u(k)=u(k-1)+\Delta u(k)
$$

The input constraint is expressed as follows:

$$
\mathbf{U}^{\min } \leq \mathbf{U} \leq \mathbf{U}^{\max }
$$

$\mathbf{U}$ can be expressed by $\Delta \mathbf{U}$ as follows:

$$
\begin{gathered}
{\left[\begin{array}{c}
u(k) \\
u(k+1) \\
u(k+2) \\
\vdots \\
u\left(k+N_{c}-1\right)
\end{array}\right]} \\
=\left[\begin{array}{c}
1 \\
1 \\
1 \\
\vdots \\
1
\end{array}\right] u(k-1)+\left[\begin{array}{ccccc}
1 & 0 & 0 & \cdots & 0 \\
1 & 1 & 0 & \cdots & 0 \\
1 & 1 & 1 & \cdots & 0 \\
\vdots & \vdots & \vdots & \ddots & \vdots \\
1 & 1 & 1 & \cdots & 1
\end{array}\right]\left[\begin{array}{c}
\Delta u(k) \\
\Delta u(k+1) \\
\Delta u(k+2) \\
\vdots \\
\Delta u\left(k+N_{c}-1\right)
\end{array}\right] .
\end{gathered}
$$

\section{Define}

$$
\begin{gathered}
\mathbf{C}_{1}=\left[\begin{array}{c}
1 \\
1 \\
1 \\
\vdots \\
1
\end{array}\right], \quad \mathbf{C}_{2}=\left[\begin{array}{ccccc}
1 & 0 & 0 & \cdots & 0 \\
1 & 1 & 0 & \cdots & 0 \\
1 & 1 & 1 & \cdots & 0 \\
\vdots & \vdots & \vdots & \ddots & \vdots \\
1 & 1 & 1 & \cdots & 1
\end{array}\right], \\
\psi=\left[\begin{array}{r}
-\mathbf{C}_{2} \\
\mathbf{C}_{2}
\end{array}\right], \quad \gamma=\left[\begin{array}{r}
-\mathbf{U}^{\min }+\mathbf{C}_{1} u(k-1) \\
\mathbf{U}^{\max }-\mathbf{C}_{1} u(k-1)
\end{array}\right],
\end{gathered}
$$

and constraint (23) can be rewritten as follows:

$$
\psi \Delta \mathbf{U} \leq \gamma
$$

Hence, the constrained optimization problem can be described as

$$
\begin{array}{ll}
\min & J=\left\|\mathbf{Y}-\mathbf{r}_{s}\right\|_{\mathbf{Q}}^{2}+\|\Delta \mathbf{U}\|_{\mathbf{R}}^{2} \\
\text { s.t. } & \psi \Delta \mathbf{U} \leq \gamma
\end{array}
$$

which can be solved by quadratic programming (QP) method.

4.2. The Nonlinear Fuzzy MPC. Based on the T-S fuzzy model, the controller is devised via the parallel distributed control (PDC) scheme $[19,20]$. In this scheme, the overall controller, which is naturally nonlinear, is a fuzzy combination of each individual linear controller designed based on the corresponding local model. Here, a nonlinear MPC controller, consisting of nine local MPC controllers, is constituted as shown in Figure 5. For each local linear model, a conventional MPC controller is designed independently. 
TABLE 3: The parameters of the nine local controllers.

\begin{tabular}{|c|c|c|c|c|c|c|c|c|c|}
\hline \multirow{2}{*}{ Parameters } & \multicolumn{9}{|c|}{$i_{\text {th }}$ controller } \\
\hline & 1 & 2 & 3 & 4 & 5 & 6 & 7 & 8 & 9 \\
\hline$q$ & 1 & 1 & 1 & 1 & 1 & 1 & 1 & 1 & 1 \\
\hline$r$ & 0.07 & 0.01 & 0.02 & 0.07 & 0.1 & 0.1 & 0.1 & 0.05 & 0.1 \\
\hline$N_{p}$ & 380 & 430 & 400 & 340 & 290 & 300 & 325 & 400 & 460 \\
\hline$N_{c}$ & 50 & 45 & 45 & 50 & 60 & 60 & 60 & 40 & 30 \\
\hline
\end{tabular}

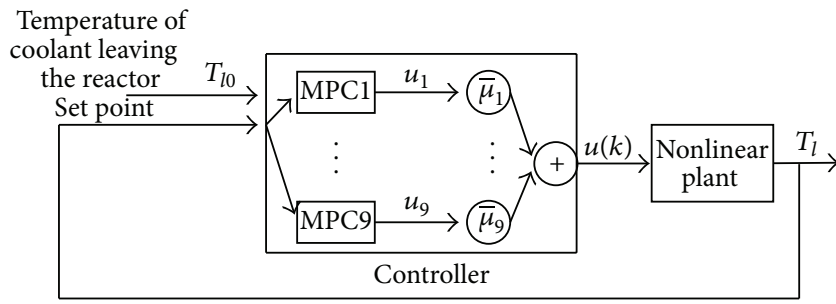

FIGURE 5: The schematic diagram of fuzzy MPC system.

Consequently, the final control is the weighted sum of the control obtained from nine local MPC controllers. Consider the following:

$$
u(k)=\sum_{i=1}^{9} \bar{\mu}_{i} u_{i}(k),
$$

where $u_{i}(k)$ is the control of the $i$ th local predictive controller and $\bar{\mu}_{i}$ is defined previously in (11). Since fuzzy logics dominate the switching between local controllers, the resulting control can be smooth by properly selecting the weights $\bar{\mu}_{i}$ [10].

\section{The Application to the PWR Nuclear Power Plant}

In using the proposed fuzzy MPC, the reference temperature function of power level is defined as follows:

$$
T_{l 0}=290+24.51 n_{r 0} .
$$

Figure 6 shows this relationship.

Assume that the maximum speed of control rod is 0.2 fraction of core length per second. Hence, the constraint on the control is considered as $-0.2 \leq u \leq 0.2$ which is involved in the optimization algorithm of MPC.

Define

$$
\mathbf{Q}=q \times\left[\begin{array}{cccc}
\mathbf{I}_{2 \times 2} & \mathbf{0} & \cdots & \mathbf{0} \\
\mathbf{0} & \mathbf{I}_{2 \times 2} & \cdots & \mathbf{0} \\
\vdots & \vdots & \ddots & \vdots \\
\mathbf{0} & \mathbf{0} & \cdots & \mathbf{I}_{2 \times 2}
\end{array}\right]_{N_{p} \times N_{p}}
$$

and $\mathbf{R}=r \times \mathbf{I}_{N_{c} \times N_{c}}$, where $q$ and $r$ are the two coefficients. Assume the control rod worth $G_{r}$ to be 0.0145 , the parameters of the nine local controllers are listed in Table 3.

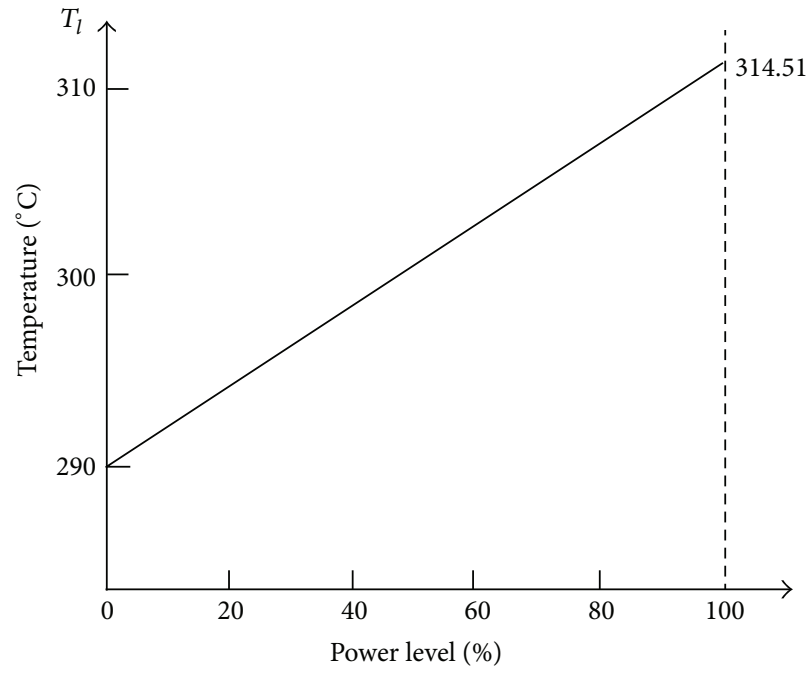

FIGURE 6: Reference temperature function of power level.

The performance of the nonlinear fuzzy MPC can be evaluated in three cases.

Case A. Full power operation, that is, $100 \% \rightarrow 90 \% \rightarrow$ $100 \%$ step changes in power level. Comparison of the performance between the proposed fuzzy MPC and the conventional PID controller is presented in Figures 7(a) and 7(b). The temperature and the power stabilize to their respect set points in an acceptable time, whereas the nonlinear fuzzy MPC reduces the fluctuation obviously. In real-time nuclear power control, inserting the control rod can slow down the release of energy in the reactor so that the temperature and power level are decreased, while lifting the control rod speed will raise the temperature and power level.

Case B. Low power operation, that is, $20 \% \rightarrow 10 \% \rightarrow 20 \%$ step changes in power level. Comparison of the performance between the proposed controller and the conventional PID controller is presented in Figures 8(a) and 8(b), showing the obvious advantage of the proposed fuzzy MPC. It is noticed that the response time is longer than that in the full power operation and the resulting fluctuation is more severe.

In both cases, the constraint on the control rod speed can be handled by the proposed fuzzy MPC effectively, while the PID controller cannot achieve it, as shown in Figures 7(c) and $8(c)$. 


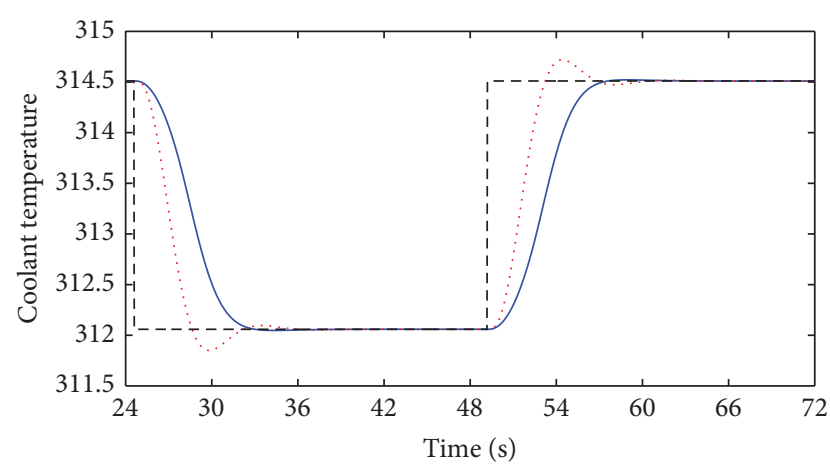

— Nonlinear fuzzy MPC --- Reference trajectory PID

(a)

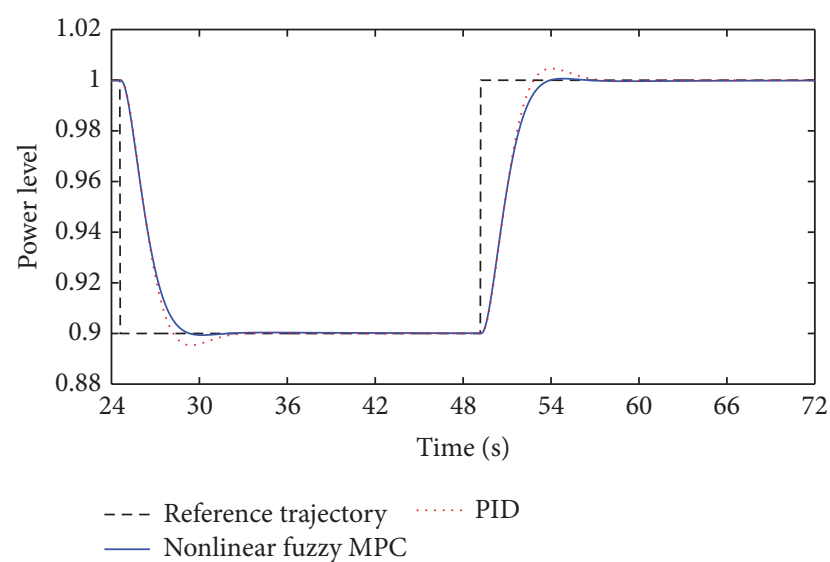

(b)

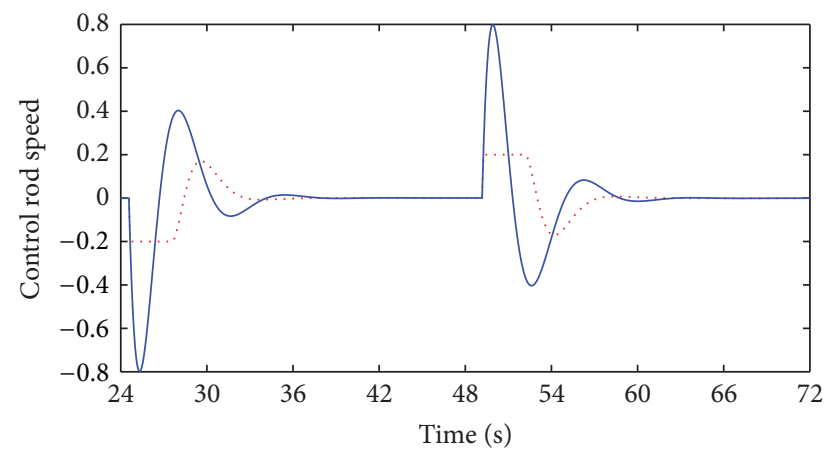

Nonlinear fuzzy MPC

- PID

(c)

Figure 7: Comparison of the performance between the nonlinear fuzzy MPC and the conventional PID controller $(100 \% \rightarrow 90 \% \rightarrow$ $100 \%$ power level change), (a) coolant temperature (unit: ${ }^{\circ} \mathrm{C}$ ), (b) power level, and (c) control rod speed.

Case C. Wide-range load following, that is, $100 \% \rightarrow$ $10 \% \rightarrow 100 \%$ ramp changes in power level with $5 \% / \mathrm{min}$ rate, as shown in Figure 9. With the constraint handling on the control rod speed by the fuzzy MPC, satisfactory tracking performance is obtained for both temperature and power.

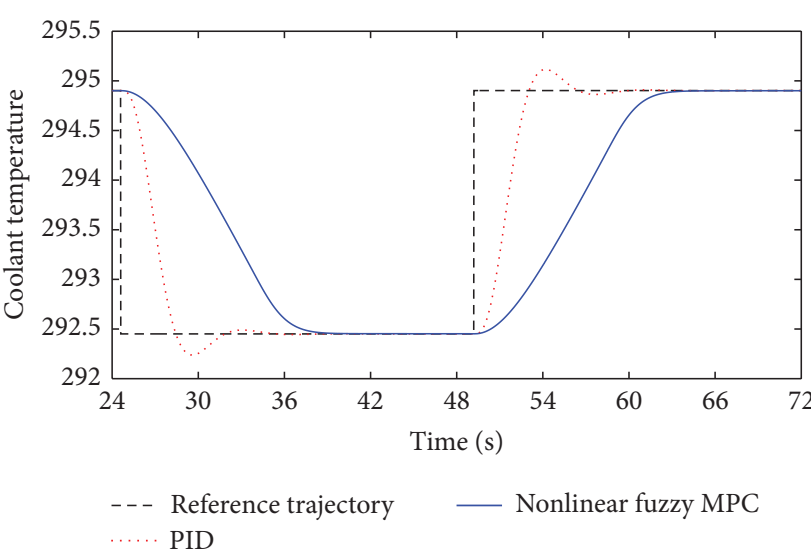

(a)

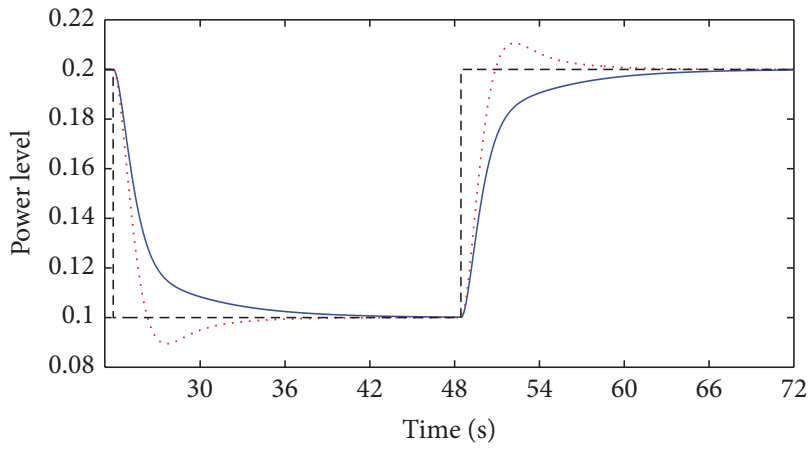

— Nonlinear fuzzy MPC --- Reference trajectory PID

(b)

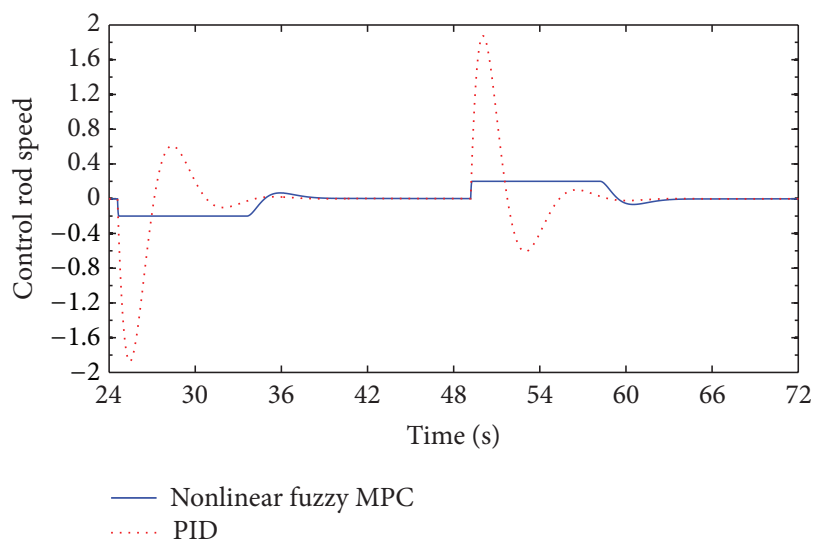

(c)

FIGURE 8: Comparison of the performance between the nonlinear fuzzy controller and the conventional PID controller $(20 \% \rightarrow$ $10 \% \rightarrow 20 \%$ power level change), (a) coolant temperature (unit: ${ }^{\circ} \mathrm{C}$ ), (b) power level, and (c) control rod speed.

\section{Conclusions}

The paper constituted a nonlinear MPC controller by incorporating fuzzy modeling technique. Feasible optimal solutions have been acquired under plant nonlinearity and constraints. The proposed nonlinear fuzzy MPC is simulated in power and temperature control for a three-mile island- 


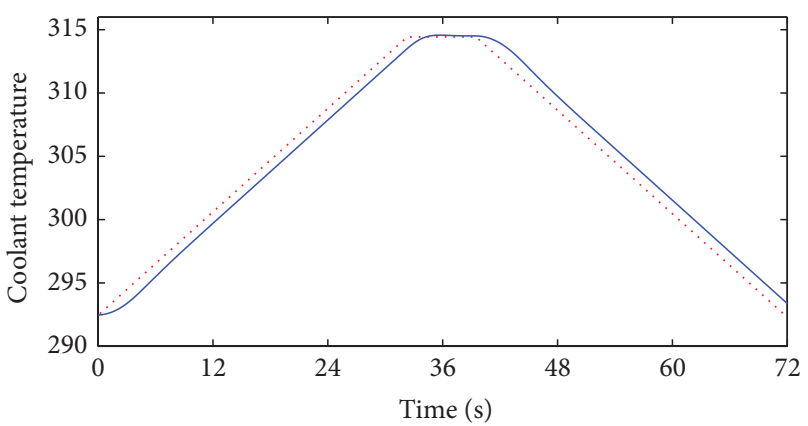

… Reference trajectory - Coolant temperature

(a)

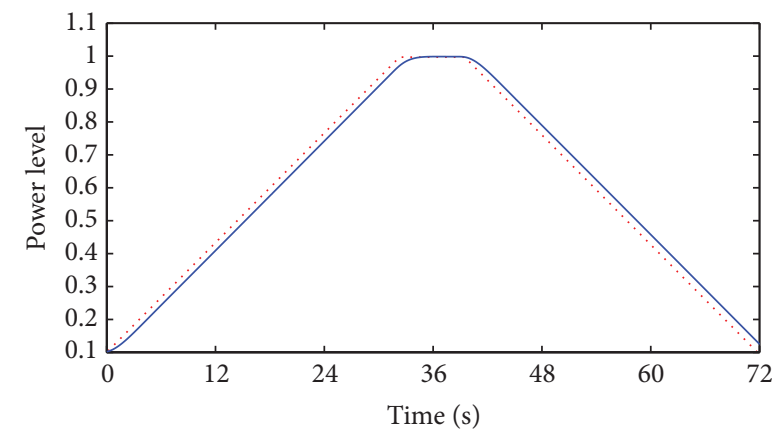

... Reference trajectory

- Power level

(b)

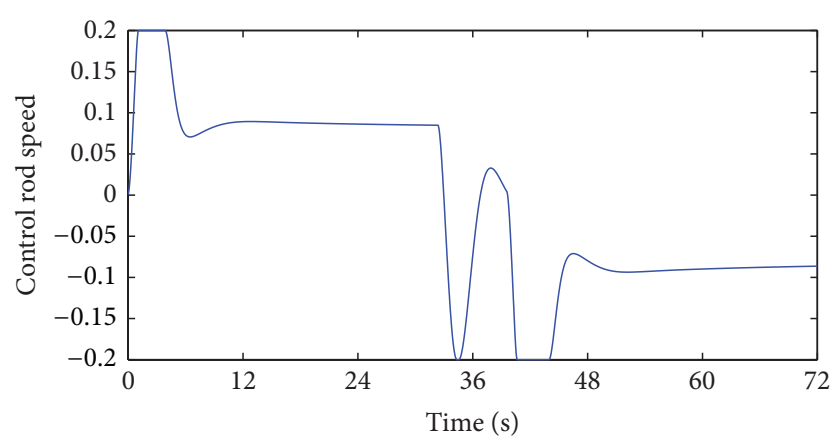

(c)

FIGURE 9: The performance for the nonlinear fuzzy controller $(100 \% \rightarrow 10 \% \rightarrow 100 \%$ ramp changes in power level with $5 \% / \mathrm{min}$ rate), (a) coolant temperature (unit: ${ }^{\circ} \mathrm{C}$ ), (b) power level, and (c) control rod speed.

(TMI-) type PWR. Better performance is obtained while it is compared with the PID controller. Load following is accomplished under constraints on the control rod.

\section{Nomenclature}

$n_{r}$ : Neutron density relative to density at rated condition

$c_{r}$ : Precursor density relative to density at rated condition $\delta \rho:$ Reactivity

$\delta \rho_{r}$ : Reactivity due to the control rod

$\beta$ : Fraction of delayed fission neutrons

$\Lambda$ : Effective prompt neutron lifetime

$\lambda$ : $\quad$ Effective precursor density

$T_{f}$ : Average reactor fuel temperature

$f_{f}$ : Fraction of reactor power deposited in fuel

$\mu_{f}$ : Heat capacity of the fuel

$P_{a 0}$ : Rated power level

$\Omega$ : Heat transfer coefficient between fuel and coolant

$T_{l}$ : The temperature of coolant leaving the reactor

$T_{e}$ : The temperature of coolant entering the reactor

$\mu_{c}$ : Heat capacity of the coolant

$M$ : Mass flow rate times heat capacity of the water

$G_{r}$ : Total reactivity worth of the rod

$z_{r}$ : The control rod speed (fraction of core length per second)

$\alpha_{f}$ : Fuel temperature reactivity coefficient

$\alpha_{c}$ : Coolant temperature reactivity coefficient.

\section{Conflict of Interests}

The authors declare that there is no conflict of interests regarding the publication of this paper.

\section{Acknowledgments}

This work was supported by National Natural Science Foundation of China under Grants nos. 60974051 and 61273144 and by Natural Science Foundation of Beijing under Grant no. 4122071 .

\section{References}

[1] R. M. Edwards, K. Y. Lee, and A. Ray, "Robust optimal control of nuclear reactors and power plants," Nuclear Technology, vol. 98, no. 2, pp. 137-148, 1992.

[2] R. M. Edwards, K. Y. Lee, and M. A. Schultz, "State feedback assisted classical control: an incremental approach to control modernization of existing and future nuclear reactors and power plants," Nuclear Technology, vol. 92, no. 2, pp. 167-185, 1990.

[3] H. Arab-Alibeik and S. Setayeshi, "Improved temperature control of a PWR nuclear reactor using an LQG/LTR based controller," IEEE Transactions on Nuclear Science, vol. 50, no. 1, pp. 211218, 2003.

[4] A. Ben-Abdennour, R. M. Edwards, and K. Y. Lee, "LQG/LTR robust control of nuclear reactors with improved temperature performance," IEEE Transactions on Nuclear Science, vol. 39, no. 6, pp. 2286-2294, 1992.

[5] Z. Dong, J. Feng, and X. Huang, "Nonlinear observer-based feedback dissipation load-following control for nuclear reactors," IEEE Transactions on Nuclear Science, vol. 56, no. 1, pp. 272-285, 2009. 
[6] H. Eliasi, M. B. Menhaj, and H. Davilu, "Robust nonlinear model predictive control for a PWR nuclear power plant," Progress in Nuclear Energy, vol. 54, no. 1, pp. 177-185, 2012.

[7] C.-C. Ku, K. Y. Lee, and R. M. Edwards, "Improved nuclear reactor temperature control using diagonal recurrent neural networks," IEEE Transactions on Nuclear Science, vol. 39, no. 6, pp. 2298-2308, 1992.

[8] P. Ramaswamy, R. M. Edwards, and K. Y. Lee, "Fuzzy logic controller for nuclear power plant," in Proceedings of the 2nd International Forum on Applications of Neural Networks to Power Systems (ANNP '93), pp. 29-34, Yokohama, Japan, 1993.

[9] X. J. Liu, P. Guan, and C. W. Chan, "Nonlinear multivariable power plant coordinate control by constrained predictive scheme," IEEE Transactions on Control Systems Technology, vol. 18, no. 5, pp. 1116-1125, 2010.

[10] X.-J. Liu and C. W. Chan, "Neuro-fuzzy generalized predictive control of boiler steam temperature," IEEE Transactions on Energy Conversion, vol. 21, no. 4, pp. 900-908, 2006.

[11] X. J. Liu and X. B. Kong, "Nonlinear fuzzy model predictive iterative learning control for drum-type boiler-turbine system," Journal of Process Control, vol. 23, no. 8, pp. 1023-1040, 2013.

[12] M. G. Na, "Auto-tuned PID controller using a model predictive control method for the steam generator water level," IEEE Transactions on Nuclear Science, vol. 48, no. 5, pp. 1664-1671, 2001.

[13] M. G. Na, Y. R. Sim, and Y. J. Lee, "Design of an adaptive predictive controller for steam generators," IEEE Transactions on Nuclear Science, vol. 50, no. 2, pp. 186-193, 2003.

[14] M. V. Kothare, B. Mettler, M. Morari, P. Bendotti, and C.-M. Falinower, "Level control in the steam generator of a nuclear power plant," IEEE Transactions on Control Systems Technology, vol. 8, no. 1, pp. 55-69, 2000.

[15] M. G. Na, I. J. Hwang, and Y. J. Lee, "Design of a fuzzy model predictive power controller for pressurized water reactors," IEEE Transactions on Nuclear Science, vol. 53, no. 3, pp. 15041514, 2006.

[16] M. G. Na, S. H. Shin, and W. C. Kim, "A model predictive controller for nuclear reactor power," Journal of the Korean Nuclear Society, vol. 35, no. 5, pp. 399-411, 2003.

[17] R. M. Edwards, K. Y. Lee, and A. Ray, "Robust optimal control of nuclear reactors and power plants," Nuclear Technology, vol. 98, no. 2, pp. 137-148, 1991.

[18] J. Shi, J. Wang, and Y. Wang, "T-S fuzzy modeling for predicting chaotic time series based on unscented Kalman filter approach," in Proceedings of the 8th International Conference on Fuzzy Systems and Knowledge Discovery (FSKD '11), pp. 722-726, Shanghai, China, July 2011.

[19] Z. Zhang and Z. Sun, "T-S fuzzy model in discrete form and its application to identification of a plane manipulator with two degree of freedom," in Proceedings of the IEEE International Conference on Intelligent Processing Systems (ICIPS '97), vol. 1, pp. 368-372, Beijing, China, October 1997.

[20] X. Luan, A. Young, W. Han, and Y. Zhai, "Load-following control of nuclear reactors based on Takagi-Sugeno fuzzy model," in Proceedings of the 18th IFAC World Congress, pp. 8253-8258, Milano, Italy, September 2011. 


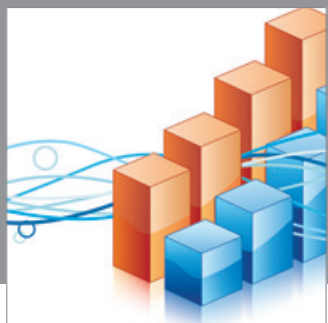

Advances in

Operations Research

mansans

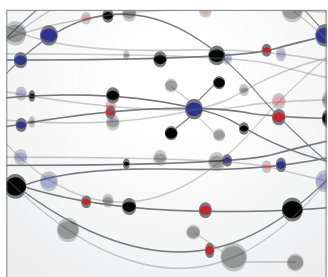

The Scientific World Journal
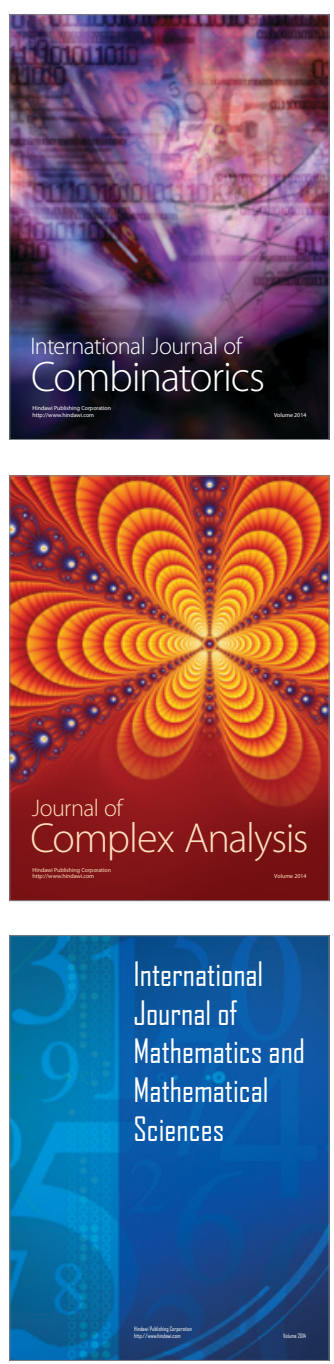
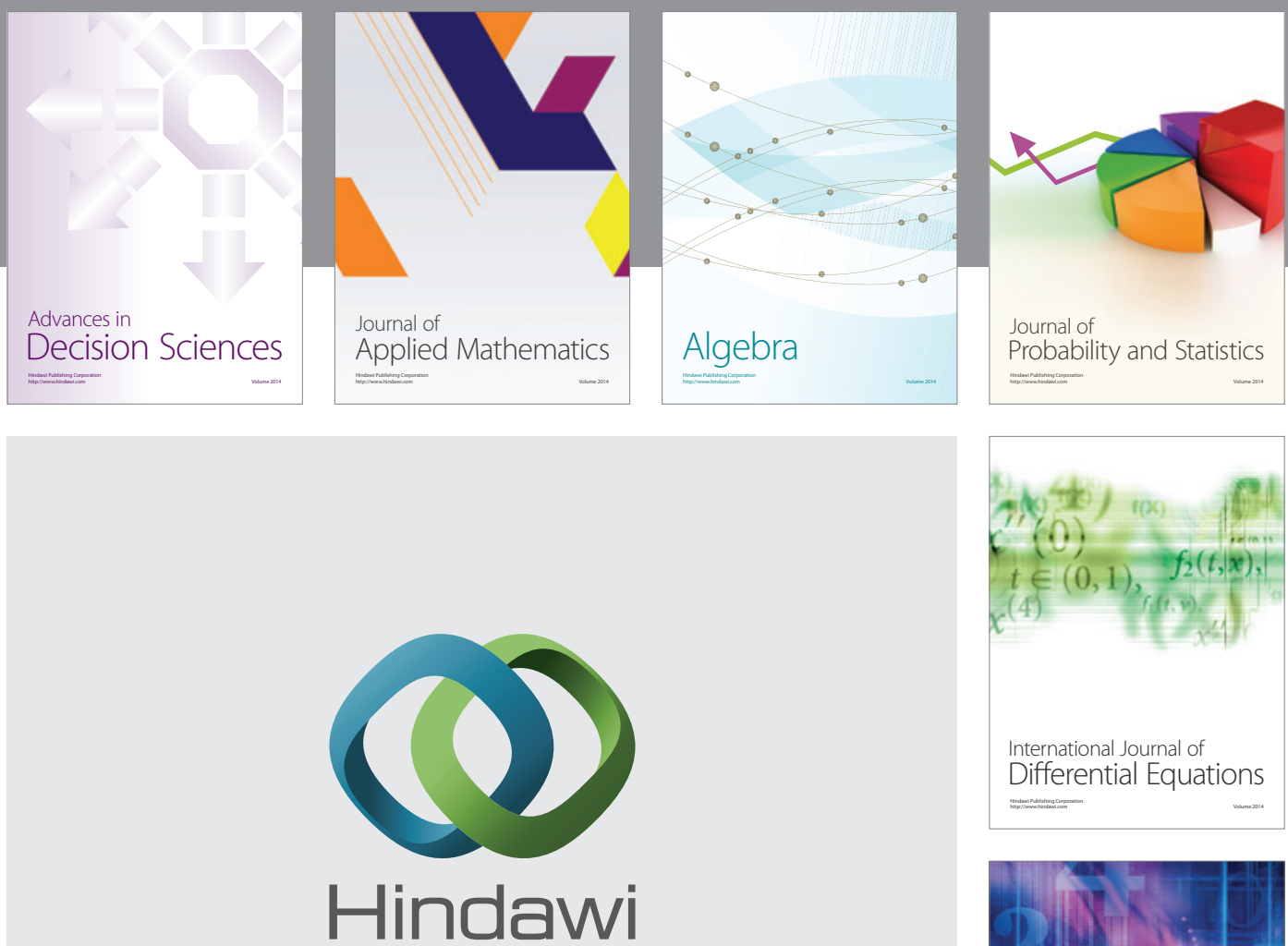

Submit your manuscripts at http://www.hindawi.com
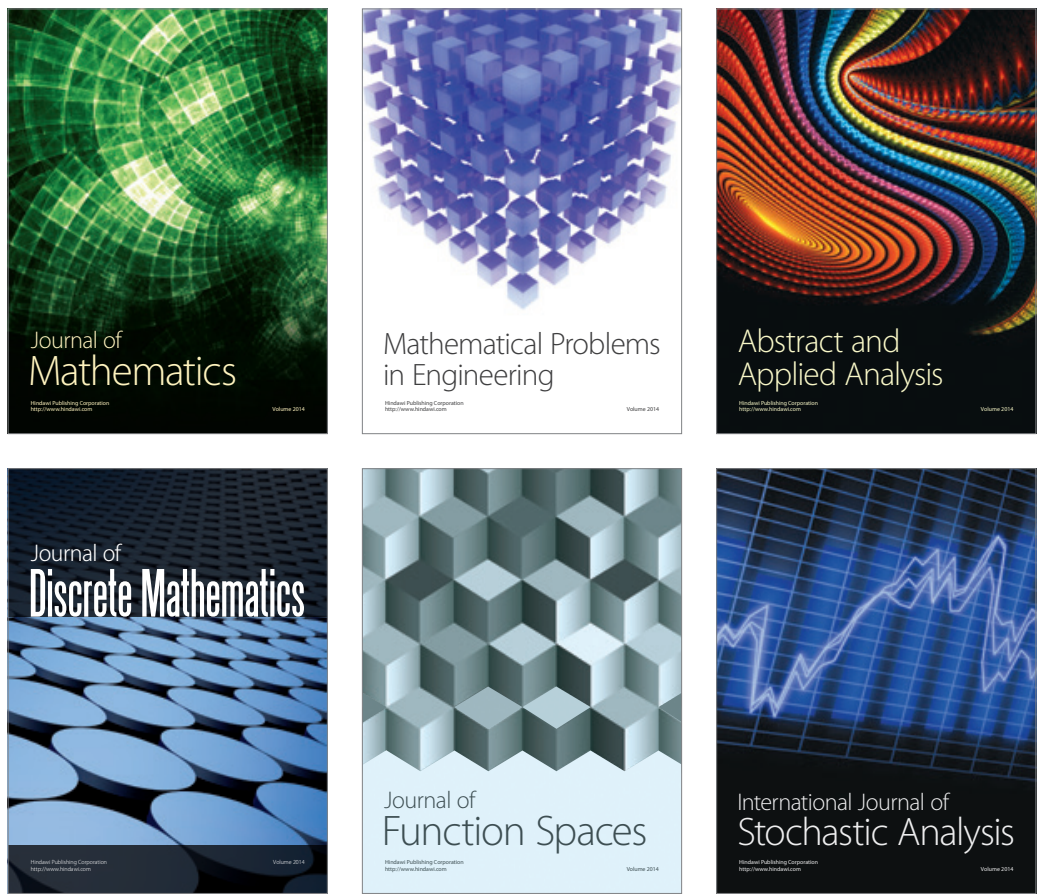

Journal of

Function Spaces

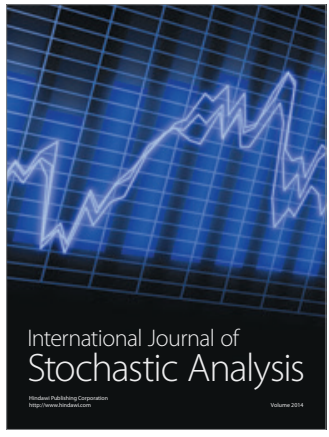

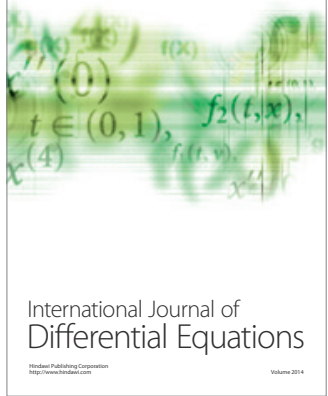
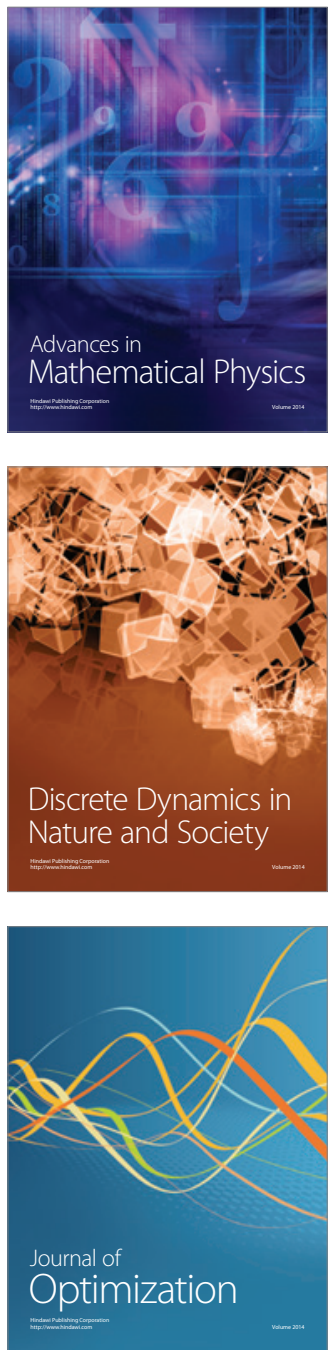